\title{
Rechtsgeschichte
}

http://www.rg-rechtsgeschichte.de/rg19

Zitiervorschlag: Rechtsgeschichte Rg 19 (2011)

$\operatorname{Rg} 192011 \quad 37-51$

http://dx.doi.org/10.12946/rg19/037-051

\section{Peter Collin}

\section{Im Auftrag der Kasse}

Überlegungen zum Gestaltwandel verwaltungsrechtsdogmatischer Konstruktionen und zu dessen rechtshistorischer Analyse 


\section{Im Auftrag der Kasse}

Überlegungen zum Gestaltwandel verwaltungsrechtsdogmatischer Konstruktionen und zu dessen rechtshistorischer Analyse

I. $\quad 33^{1 / 2}$ Flaschen Champagner und 48 Flaschen Wein

Eigentlich war das Reichsgesundheitsamt für diese Angelegenheit nicht zuständig. Die Abgabe an die zuständige Landesbehörde hätte ausgereicht. Eine derart »rücksichtslose Ausnutzung (der) Befugnisse « eines Kassenarztes, wie sie hier - im Jahr I 886 - zu Tage trat, veranlasste Gesundheitsamtsdirektor Köhler aber dann doch, den Fall Innenstaatssekretär Boetticher vorzulegen: Der Arzt hatte einer an Herzschwäche und Lungenentzündung leidenden

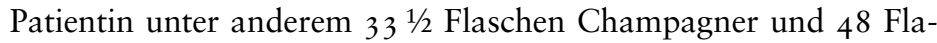
schen Wein verschrieben. Lieferung erfolgte obendrein durch den Bruder des Arztes, einen Gastwirt. ${ }^{\mathbf{I}}$ Die Krankenkasse verweigerte die Zahlung. Der Gastwirt verklagte daraufhin die Kasse. Das Landgericht Freiburg i. Br. entschied gegen die Kasse. Die Forderung des Wirts sei durch das Rezept des Arztes gedeckt. Letzterer stehe als Kassenarzt zur Kasse im Verhältnis »eines von der Kasse Beauftragten «; mit der Verschreibung der Alkoholika, die auch von einem Gutachter als taugliche Arznei bewertet wurde, habe er sich in den Grenzen seiner Vollmacht bewegt und konnte die Kasse gegenüber dem Wirt auch wirksam verpflichten. Zur Untermauerung seiner Rechtsansicht verwies das Gericht u. a. auf die achte Auflage von Puchtas Pandekten, $\mathbb{\int} 324 .^{2}$

In der Tat, was Puchta an der bezeichneten Stelle beschrieb, war der Auftrag (das Mandat), an sich also eine unentgeltliche Geschäftsbesorgung, ${ }^{3}$ in diesem Fall allerdings integriert in einen Dienstvertrag. So wurde die Rechtslage, wie sie nach Erlass des Krankenversicherungsgesetzes von I 883 bestand, auch vom späteren Schrifttum dargestellt. ${ }^{4}$ Aber war damit das Verhältnis des Kassenarztes zur Kasse zutreffend beschrieben? Ließ sich dieses hinreichend durch Privatrecht erfassen? Denn der Arzt wirkte nicht nur in einem privatrechtlichen, sondern auch in einem öffentlich-rechtlichen Pflichtenkreis. $\$ 6$ Abs. I Ziffer I des Krankenversicherungsgesetzes von I 883 verpflichtete die Krankenkassen zur

I Schreiben des Direktors des Kaiserlichen Gesundheitsamts Karl Köhler an den Staatssekretär des Innern Karl Heinrich von Boetticher vom I4. Mai I886, abgedr. in: Quellensammlung zur Geschichte der deutschen Sozialpolitik I 867 bis I9I4, II. Abt. 5. Bd., Darmstadt 2009, Nr. I05.

2 Entscheidung des Landgerichts Freiburg i. Br. vom I4. Dezember
I 887, abgedr. in: Quellensammlung (Fn. I) Nr. I 25.

3 G. F. Puchta, Pandekten, 8. Aufl., Leipzig I $856, \mathbb{S} 324$ (482 ff.).

4 A. Hess, R. Venter, Das Gesetz über Kassenarztrecht, Bd. I, Köln I95 5, I9 f.; D. Klette, Die Kassenarztverträge der sozialen Krankenversicherung. Ihr geschichtlicher Werdegang mit Motiven, Diss. jur. Tübingen I 965 , 28, 38 . 
5 Gesetz, betreffend die Krankenversicherung der Arbeiter, vom I 5. Juni I 883 (RGBl. S. 73). $\$ 6$ regelte nur die Leistungsverpflichtungen der Gemeindekrankenversicherung, die Vorschriften zu den einzelnen Krankenkassentypen nahmen jedoch auf diese Vorschrift Bezug (siehe z. B. für die Ortskrankenkassen $\mathbb{2} 2$ Abs. I Ziffer I).

6 So auch noch heute F. E. Schnapp, Muss ein Vertragsarzt demokratisch legitimiert sein?, in: Neue Zeitschrift für Sozialrecht 200I, 337-34I, 337.

7 Th. Tauchnitz, Die »organisierte" Gesundheit. Entstehung und Funktionsweise des Netzwerks aus Krankenkassen und Ärzteorganisationen im ambulanten Sektor, Wiesbaden 2004, I06 f.

8 Gemeint sind hier die Orts-, Betriebs-, Bau- und Innungskrankenkassen nach den $\mathbb{S} \mathbb{1} 6 \mathrm{ff}$. Krankenversicherungsgesetz I 883 (Fn. 5), nicht der verbliebene Restbestand an privatrechtlichen Kassenvereinen; siehe dazu P. Collin, Optionen und Kontinuitäten regulierter Selbstregulierung in der frühen Krankenund Altersversicherung, in: $\mathrm{Rg}$ I 8 (2OII) I 86-2I2, I99 ff.

9 H. Rosin, Das Recht der öffentlichen Genossenschaft, Freiburg I $886,62 \mathrm{ff}$.

Io Rosin, Genossenschaft (Fn. 9) I8. Allerdings ist $\mathrm{zu}$ unterscheiden: Insofern $\ 6$ Krankenversicherungsgesetz der Kasse eine Aufgabe zuwies, handelte es sich um öffentlich-rechtliches Organisationsrecht. Daraus resultierte aber kein öffentlich-rechtlicher Behandlungsanspruch des Versicherten. Dessen Verhältnis zur Krankenkasse war nach der nach I 883 herrschenden Auffassung privatrechtlicher Natur; siehe Entscheidung des OLG Dresden vom 24. Februar I 886, Quellensammlung (Fn. I) Nr. IOI (477).

I I Gängige Praxis war, dass die Kassenarztstellen ausgeschrieben wurden und der Kassenvorstand über die Auswahl der Bewerber entschied, siehe z. B. Quellensammlung (Fn. I) Nr. I 44.

I2 Siehe E. v. WoedtKe, Krankenversicherungsgesetz, 3. Aufl., Berlin, Leipzig I 886, 68, der ebenfalls

Gewährung der Krankenunterstützung in Form ärztlicher Behandlung und der Verschreibung und Vergabe von Arzneimitteln. ${ }^{5}$ Der Kassenarzt realisierte, besser: konkretisierte, die öffentlich-rechtliche Pflicht der Krankenkassen. ${ }^{6}$ Er behandelte nicht nur den Patienten, er entschied durch seine Expertise auch darüber, ob und wie diese Pflicht zu erfüllen war. ${ }^{7}$ Dass es sich um eine Verpflichtung öffentlich-rechtlicher Natur handelte, dürfte spätestens nach der I 886 erschienenen Monographie von Rosin über die öffentlichen Genossenschaften unumstritten gewesen sein. Die Krankenkassen ${ }^{8}$ waren öffentlich-rechtliche Genossenschaften, ${ }^{9}$ die sich gerade dadurch definierten, dass ihnen eine öffentlichrechtliche Pflicht, hier die Krankenversorgung, auferlegt war. ${ }^{\text {Io }}$

Zur Integration des Kassenarztes in die öffentlich-rechtliche Pflichterfüllung traf das Krankenversicherungsgesetz allerdings keine Aussagen; ${ }^{\text {II }}$ die frühen Kommentierungen enthielten keine darüber hinausgehenden Ausführungen. ${ }^{\text {I2 }}$ Man könnte es bei dem Befund belassen, dass die Inanspruchnahme des Arztes privatrechtlich erfolgte. Legte aber nicht seine Einbindung in einen öffentlich-rechtlichen Pflichtenkreis öffentlich-rechtliche Statusbeschreibungen und die Anerkennung öffentlich-rechtlicher Ansprüche und Bindungen nahe?

Am Beispiel des Kassenarztes gehen die folgenden Ausführungen der Frage nach, auf welchen Wegen sich die begriffliche und systematische Erfassung gemeinwohlrelevanter Tätigkeitskomplexe vollzog. Zunächst soll skizziert werden, welche rechtsdogmatischen Konstruktionen mit übergreifender Geltungsreichweite für die hoheitliche Inanspruchnahme Privater ab dem Ende des I9. Jahrhunderts entwickelt wurden. Damit wird der Horizont ausgeleuchtet, in den sich die Genese der Konstruktion des Kassenarztstatus einbetten lässt.

\section{Indienstnahme Privater für öffentliche Aufgaben: Pfade der Begriffsentwicklung}

Sowohl die konstitutionelle Staatsrechtslehre als auch die sich als eigenständige Disziplin etablierende Verwaltungsrechtswissenschaft erkannten dem Grunde nach die Zulässigkeit der Übertragung öffentlicher Funktionen an. ${ }^{\mathbf{I}}$ Dem staatlichen Regelungsund Vollzugsmonopol tat man damit keine Gewalt an, handelte es

lediglich auf die Auswahl des Arztes durch die Kasse hinweist, darüber hinaus aber keine Aussagen zur Stellung des Kassenarztes trifft.

I3 Siehe z. B. L. v. Rönne, Das Staats-Recht der Preußischen Monarchie, Bd. I, I. Abt., 3. Aufl. Leipzig I 869, 2I 5; G. MEYER, Lehrbuch des Deutschen Verwaltungsrechts, Teil I, Leipzig I $88_{3}$, I 8 f.; C. Gareis, Allgemeines Staatsrecht (Handbuch des Öf- fentlichen Rechts, Bd. I, Hbd. I), Freiburg, Tübingen I $88_{3}, 86$ ff.; O. v. SARWEY, Allgemeines Verwaltungsrecht (Handbuch des Öffentlichen Rechts, Bd. I, Hbd. 2), Freiburg, Tübingen I 884, 95; E. Loening, Lehrbuch des Deutschen Verwaltungsrechts, Leipzig I 884, 9 f.; A. ARndT, Das Verordnungsrecht des Deutschen Reichs, Berlin, Leipzig I 884, 24; G. Jellinek, System der subjekti- 
sich doch um delegierte und nicht um originäre Befugnisse der Privaten. In welchem Ausmaß und für welche Fälle eine Aufgabenübertragung zulässig war, darüber gingen teilweise die Auffassungen auseinander, teilweise ließen sich den meist knappen Stellungnahmen keine dahingehenden Aussagen entnehmen. Es handelte sich nicht um ein Zentralthema. Nur soviel stand fest, dass als Übertragungsinstrumente sowohl privatrechtliche, v. a. der Dienstvertrag, als auch öffentlich-rechtliche Modi in Betracht kamen. Dieser Befund gilt im Grunde auch für das Schrifttum der Weimarer Zeit. ${ }^{{ }^{14}}$ Strikt ablehnende Haltungen finden sich nur selten. ${ }^{{ }^{5}}$ Wie aber ließen sich solche Varianten der Aufgabenwahrnehmung rechtsinstitutionell fassen und in ein verwaltungsrechtliches System integrieren? Klare Leitlinien sind hierbei nicht erkennbar. Der Versuch, sie im etablierten dogmatischen Instrumentarium unterzubringen, indem man mit öffentlichen Aufgaben versehenen Privaten den Status einer »teilweise " öffentlich-rechtlichen Person zuerkannte, ${ }^{\mathbf{1}}$ stieß jedenfalls nicht auf breite Anerkennung (auch wenn er damit den Weg zum [modernen] funktionellen bzw. relativen Behördenbegriff ${ }^{17}$ des Verwaltungsverfahrensrechts wies)..$^{18}$

Es handelte sich um ein recht vielgestaltiges Phänomen, das so unterschiedliche Fall- bzw. Personengruppen wie die Beauftragung von Privatbahnen mit der Bahnpolizeigewalt, den Börsenmäkler, den Bezirksschornsteinfeger, den Fährmann, den Privatdozenten, den bei der Brandbekämpfung helfenden Bürger oder den technischen Überwachungsverein einschloss. Das geltende Recht bot keine Anhaltspunkte für verallgemeinerbare Aussagen. Das gemeine Recht (bzw. später das BGB) stellte nur die privatrechtlichen Rechtsfiguren zur Verfügung, die sich in privatrechtlichen Verhältnissen zum Einsatz bringen konnten. Das Allgemeine Landrecht sah aufbauorganisatorische Formen vor, die Korporationen, ${ }^{19}$ die aber die Verleihung des Korporationsstatus voraussetzten und nur für kollektive Akteure, nicht für Einzelpersonen in Betracht kamen. Die Aufopferungsregeln der $\mathbb{S} \mathbb{5 4}$, 75 Einl. ALR kamen nur für einzelne Konstellationen erzwungener Inanspruchnahme in Frage. Man betrat also Neuland.

Die begrifflich folgenreichste Konstruktion stammt von Otto Mayer, denn das von ihm aus dem Rechtsstoff herausgearbeitete Institut der "Verleihung " und des "Beliehenen " etablierte sich in terminologischer Hinsicht (»Beleihung «) als Standardmodus der Betrauung Privater mit hoheitlichen Funktionen. Jedoch zielte

ven öffentlichen Rechte, Freiburg i. Br. I $892,234 \mathrm{ff}$.

I4 Siehe nur E. Kaufmann, Art. »Verwaltung, Verwaltungsrecht «, in: Wörterbuch des Deutschen Staats- und Verwaltungsrechts, hg. von K. Freiherr v. Stengel, M. Fleischmann, Bd. 3, 2. Aufl. Tübingen I9I4, 688-7I8, 7 I5.

I 5 A. Köttgen, Rezension zu

G. Leibholz, Das Wesen der Repräsentation unter besonderer
Berücksichtigung des Repräsentativsystems, in: AöR 58 (I930) 290-3 I2, 304 .

I6 L. RiCHTER, Das subjektive öffentliche Recht, in: AöR 47 (I925) I-84, 2 I f.

I7 Siehe dazu nur H. Maurer, Allgemeines Verwaltungsrecht,

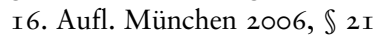
Rn. $32 \mathrm{f}$.

I 8 Zudem entsprang der dogmatische Ansatz von Richter, Das subjek- tive öffentliche Recht (Fn. I6) auch nicht dem Bestreben, ein Rechtsinstitut für dieses Phänomen zu kreieren, sondern dem Versuch, dem Problem aus dem Weg zu gehen, dass die Geltung des öffentlichen Rechts sich nicht auf die Beziehung zwischen Privaten erstrecken konnte - man musste also einem privaten Aufgabenträger, der in Erfüllung seiner Aufgaben eine Rechtsbeziehung zu einem anderen Privaten begründete, den Status der Privatrechtsperson nehmen.

I9 $\mathbb{S} 25 \mathrm{ff}$. II $6 \mathrm{ALR}$. 
Mayers rechtliche Konstruktion auf die Erfassung eines Tatbestandes, welcher nur geringe Schnittmengen mit jenem der Inanspruchnahme Privater für öffentliche Aufgaben aufwies. Unter Verleihung (Konzession) verstand Mayer einen »Verwaltungsakt, durch welchen für den Beliehenen das Recht des Besitzes an einem Stück öffentlicher Verwaltung begründet wird zur Ausübung eigenen Namens «. ${ }^{20}$ Die Umschreibung des Verleihungsobjekts als »Stück öffentlicher Verwaltung « suggeriert Aufgabenübertragung. Dass dem so nicht ist, wird deutlich, wenn man die beiden Fallgruppen der Mayer'schen Konstruktion betrachtet. Die erste betraf die Verleihung des Rechts zur besonderen Benutzung einer öffentlichen Sache für private Zwecke; ${ }^{2 I}$ als Beispiele werden genannt: Stichkanal, Anlegestelle, Badeanstalt, Privathafenanlage, Kirchstuhl, Grabstätte, Privatbücke. Dabei handelte es sich der Sache nach um eine Sondernutzungserlaubnis mit gesteigerten Rechtsgarantien. Sicher konnte - nicht musste - diese private Nutzung auch anderen als den privaten Zwecken des Beliehenen zugute kommen. Sollte dies aber in verpflichtender Weise geschehen, war hierfür der Erlass von Auflagen notwendig. ${ }^{22}$ Die Verwendung für öffentliche Zwecke war nicht essentieller Bestandteil der Verleihung. Die Verleihung des Gebrauchsrechts an öffentlichen Sachen bedeutete vielmehr die Entlassung eines Rechtsobjekts aus seiner öffentlichen Zweckbindung. Denn jenes Rechtsobjekt, welches von Mayer deshalb als »Stück öffentlicher Verwaltung « bezeichnet wurde, weil es (ursprünglich) öffentlichen Zwecken diente, ${ }^{23}$ erfuhr mit der Verleihung eine auf Privatnützigkeit zielende Umwidmung.

In stärkerem Maße erkennbar wird der Bezug zur Übertragung öffentlicher Aufgaben bei der zweiten von Mayer behandelten Unterkategorie: der »Verleihung eines öffentlichen Unternehmens «; Herstellung und Betrieb von Kunststraßen, Kanälen, vor allem aber Eisenbahnen und Telegrafenanstalten zählen hierzu. Denn dem durch die Verleihung begründeten Recht, ein öffentliches Unternehmen zu besitzen und zu betreiben, korrespondierte eine entsprechende Pflicht. Allerdings schuf sie keinen öffentlichrechtlichen organschaftlichen Status des Beliehenen, sondern veränderte lediglich seinen privatrechtlichen Status. ${ }^{24}$ Soweit einzelne Hoheitsbefugnisse mit dem Betrieb des öffentlichen Unternehmens verbunden waren, waren diese Bestandteil des Unternehmens, mit welchem sie auf den Privaten übergingen, nicht konstitutives Rechtsobjekt der Verleihung selbst. ${ }^{25}$ Dies kam schon darin zum

20 O. Mayer, Deutsches Verwaltungsrecht, Bd. 2, 2. Aufl. München, Berlin I9I7, I8I.

2I MAYER, Verwaltungsrecht (Fn. 20) I93: »er benutzt die öffentliche Sache für seinen Zweck, privatwirtschaftlich «.

22 MAYER, Verwaltungsrecht (Fn. 20) $200 \mathrm{f}$.

23 MAYER, Verwaltungsrecht (Fn. 20) 7 I.
24 Mayer, Verwaltungsrecht (Fn. 20) 459.

25 MAYer, Verwaltungsrecht (Fn. 20) 462; zur Auseinandersetzung mit der unzutreffenden Auffassung, die Mayer'sche Verleihung habe wesensnotwendig eine Übertragung von Hoheitsbefugnissen zum Gegenstand, K. Vogel, Öffentliche Wirtschaftseinheiten in privater Hand, Hamburg I959, $47 \mathrm{f}$. 
Ausdruck, dass der Beliehene grundsätzlich privatrechtlich über das Leihobjekt und damit auch über den Übergang von Hoheitsbefugnissen verfügen konnte. ${ }^{26}$

Mit dieser Konstruktion, die auf eine Rechte-, nicht auf eine Aufgabenübertragung abzielte, hatte die Mayer nachfolgende Verwaltungsrechtslehre, soweit sie sich nicht lediglich auf eine Wiederholung seines Gedankenguts ${ }^{27}$ oder dessen Einordnung in eine ausdifferenzierte Verwaltungsaktlehre ${ }^{28}$ beschränkte, jedoch gewisse Schwierigkeiten. Zum einen konnte sie nicht den Tatbestand ignorieren, dass derartige Übertragungen auch auf Verträgen gründeten. Zum Teil konzedierte man deshalb einfach, dass Verleihungen sowohl durch Verwaltungsakt als auch durch Vertrag erfolgen konnten, ${ }^{29}$ womit man allerdings die Mayer'sche Konstruktion zerstörte, denn dieser hatte mit dem Institut der Verleihung Rechtsfolgen, z. B. in Bezug auf die Aufhebung und Aufsicht, geknüpft, die sich mit einer vertraglichen Regelung nicht vereinbaren ließen; zum Teil unternahm man den Versuch, den Gesamttatbestand einer Verleihung in vertragliche und verwaltungsaktförmige Elemente aufzusplitten. ${ }^{\circ}$

Zweitens machte sich das Fehlen einer in die deutsche Rechtstradition integrierbaren Fundierung bemerkbar. Zwar war der Begriff der Verleihung nicht neu, ${ }^{3 \mathrm{I}}$ aber so wie Mayer ihn - jedenfalls in Bezug auf die öffentlichen Unternehmen - konturiert hatte, handelte es sich doch um eine dogmatische Neuschöpfung, orientiert am Modell des französischen service public, was auch von den Zeitgenossen gesehen - und abgelehnt wurde. ${ }^{32}$ Stattdessen griffen einige Autoren, wie Fleiner, auf das Institut des Regals, also eines staatlich monopolisierten Tätigkeitskomplexes, zurück. Die Verleihung stellte sich dann dar als Gestattung privatrechtlicher Übernahme dieser Tätigkeit ${ }^{33}$ - eine Verbindungslinie, die Mayer allerdings so nicht gezogen hatte: Eine Verleihung konnte, musste aber nicht eine regalrechtliche Disposition sein. ${ }^{34}$ Zudem unterfiel das Referenzmodell des Mayer'schen Instituts, die eisenbahnrechtliche Konzession, schon nach länger herrschender Meinung nicht mehr der Regalitä̈. ${ }^{35}$ Und schließlich kollidierte der (ursprünglich) privatrechtliche Charakter der regalrechtlichen Verfügung mit den deutlich erkennbaren Bemühungen, dieses Institut für die Indienstnahme Privater für öffentliche Zwecke dienstbar zu machen. Zwar konnte dies mit dem Hinweis auf einen Funktionswandel des Regals überspielt werden - nicht primär als Vermögensquelle, sondern

26 MAYER, Verwaltungsrecht (Fn. 20) 460.

27 So doch wohl F. Fleiner, Institutionen des Deutschen Verwaltungsrechts, 4. Aufl. Tübingen I9I9, 3 I $7 \mathrm{ff}$.

28 K. Kormann, System der rechtsgeschäftlichen Staatsakte, Berlin I9IO, 84 ff., IO4 ff.

29 W. JELLINEK, Verwaltungsrecht, 3. Aufl. Berlin I93 I, 527, der als Beispiele anführt: Vertrag zwi- schen dem preußischen Fiskus und Siemens \& Halske über den Betrieb von Polizeimeldeanlagen, Konzessions- und Lieferungsvertrag zwischen dem Freistaat Lippe und der Elektrizitätswerk GmbH Wesertal-Hameln über die Versorgung des Freistaats Lippe mit elektrischer Energie.

30 Hierzu K. Romberg, Die rechtliche Natur der Konzessionen und Schutzbriefe in den deutschen
Schutzgebieten, Diss. jur Berlin I909, I I f., in Bezug auf die Kolonialkonzessionen.

3 I Allgemein als üblicher Terminus: »Verleihung « eines Privilegs, weiter: »Verleihung « des Bürgerrechts, eines Ordens, des Promotionsrechts; näher am Mayer'schen Verständnis dann: »Verleihung « der Befugnis zur Erhebung von Chausseegeldern, überhaupt fiskalischer Vorrechte.

32 Besonders Kaufmann, Verwaltung (Fn. I4) $705 \mathrm{ff}$.

33 F. Fleiner, Institutionen (Fn. 27) $3 \mathrm{I} 7 \mathrm{ff}$., inbs. 32I; zuvor schon ebenso in der Sache (freilich ohne Rückgriff auf das Mayer'sche Institut der Verleihung) W. LUDEwIG, Die Regalität der Telegraphie im Allgemeinen und in Deutschland insbesondere, in: Zeitschrift für das Gesammte Handelsrecht 3I (I 885 ) 63-I 24, 69 ff., insb. 73 .

34 MAYER, Verwaltungsrecht (Fn. 20) $434 \mathrm{f}$.

35 Meyer, Verwaltungsrecht (Fn. I3) 492 ff., insb. 495, Fn. I; K. FreIHERR V. STENGEL, Lehrbuch des Verwaltungsrechts, Stuttgart I886, 426 f.; siehe auch TH. KITTEL, Die Verleihung von Schienenwegen, insbesondere von Straßenbahnen, Diss. Leipzig I $906,24 \mathrm{ff}$. 
36 O. Potschter, Wesen und Recht der Konzession (Verleihung) in Deutschland und Sowjetrußland, Jena I928, 8; i. E. auch Fleiner, Institutionen (Fn. 27) $32 \mathrm{I}$.

37 J. HATscheK, Lehrbuch des deutschen und preußischen Verwaltungsrechts, 5./6. Aufl. Leipzig I927.

38 JELLINEK, Verwaltungsrecht (Fn. 29).

39 So z. B. Jellinek, Verwaltungsrecht (Fn. 29) 526 f., damit auf die Traditionslinie des hergebrachten Konzessionsbegriffs verweisend; siehe dazu H. Mohnhaupt, Art. »Konzession ", in: Enzyklopädie der Neuzeit, Bd. 7, Darmstadt 2008, Sp. II-I4.

40 Siehe R. Arend, Monopole und Privilegien im heutigen Verwaltungsrecht, Diss. jur. Köln I93 I, I2, 34 f., der die Apothekerkonzession als Fall der Verleihung einstuft, obwohl Apotheken keine öffentlichen Unternehmungen im Sinne Mayers darstellten.

4I Kaufmann, Verwaltung (Fn. I4) 7I3; O. BüHLER, Zur Theorie des subjektiven öffentlichen Rechts, in: Festgabe für Fritz Fleiner zum 60. Geburtstag, Tübingen I927, 26-58, 37 f.; G. Meyer, G. ANsCHÜTZ, Art. »Konzessionen «, in: Wörterbuch des Staats- und Verwaltungsrechts (Fn. I4) Bd. 2, 2. Aufl. I9I3, 630-63 I; s. auch schon H. Reнm, Die rechtliche Natur der Gewerbs-Konzession, München I 889, $36 \mathrm{f}$., der eisenbahnrechtliche Konzession und

dem Gemeinwohl sollte es dienen ${ }^{36}$-, jedoch vermochte sich die regalienrechtliche Einbettung der Verleihung nicht zu halten; das Regal mit seinen vormodernen Wurzeln hatte in einer Verwaltungsrechtswissenschaft des 20. Jahrhunderts keine Chance mehr; bei Hatschek $^{37}$ und Jellinek ${ }^{38}$ tauchte es im Zusammenhang mit der Verleihung nicht mehr auf.

Letztendlich stieß drittens die von Mayer vorgenommene Abgrenzung der Verleihung (»Konzession«) von der gewerberechtlichen Polizeierlaubnis immer weniger auf Verständnis. Sicher ließ sich rechtssystematisch differenzieren: die Gewerbeerlaubnis als gebundene Entscheidung, bei der die garantierte Gewerbefreiheit nur unter einem Kontrollvorbehalt stand, die Konzession hingegen als Gestattung eines Betriebes, auf dessen Ausübung wegen des prinzipiellen staatlichen Monopolrechts kein Anspruch bestand. ${ }^{39}$ Aber erstens ließen schon mit gesteigerten Anforderungen verbundene und nicht ohne weiteres unter den gewerberechtlichen Genehmigungsanspruch subsumierbare Erlaubnisse (Apothekerkonzession) die Möglichkeit einer saubere Trennung fraglich erscheinen, ${ }^{\circ}$ und zweitens zweifelte man grundsätzlich ihre Strukturverschiedenheit an und behandelte sie als einheitliche Rechtsfigur. ${ }^{4 \mathbf{I}}$ So wurde zwar das Rechtsinstitut der Verleihung weiter tradiert, hatte aber an innerer Konsistenz verloren. Hatschek und Huber verlagerten den Schwerpunkt auf die »Konzession von Hoheitsrechten « - bei Mayer nur ein Annex - und rekurrierten damit nicht mehr auf das ursprüngliche konstitutive Element der Unternehmensverleihung, ${ }^{42}$ Richter übernahm von Mayer zwar die Formulierung von der Übertragung »eines Stücks öffentlicher Verwaltung «, leitete daraus aber - wie schon erwähnt - eine Statusänderung des Beliehenen ab: Dieser werde »teilweise « zu einer öffentlich-rechtlichen Person. ${ }^{43}$ Die rechtskonstruktive Verbindung zum Mayer'schen Institut der Verleihung war damit abgerissen.

Nach dem Krieg war der Bezug zwar noch gegenwärtig, aber die Distanz zur Unternehmensverleihung und die rechtsdogmatische Unterbilanz ${ }^{44}$ wurden nun offen ausgesprochen, und es wurden - teilweise in Anknüpfung an in der Weimarer Zeit entwickelte Gedanken (Hatschek, Huber) - die Konturen des heutigen Beleihungsrechts entwickelt, ${ }^{45}$ bis sie in der bis heute unerreichten rechtssystematischen Darstellung des Verwaltungsorganisationsrechts von Wolff/ (Bachof) ${ }^{46}$ ihre Ausformung und allgemeine Anerkennung fanden. Im Ergebnis wurde damit die Beleihung in der

Gewerbeerlaubnis in öffentlichrechtlicher Hinsicht gleichstellt; den Unterschied sieht er lediglich darin, dass die Eisenbahnkonzession eine stärkere privatrechtliche Schutzwirkung entfaltet.

42 Hatschek, Verwaltungsrecht (Fn. 37) 356; E. R. Huber, Wirtschaftsverwaltungsrecht, Tübingen I932, 47.

43 L. Richter, Das subjektive öffentliche Recht (Fn. I6) 2I f.
44 Siehe auch H.H. Rupp, Privateigentum an Staatsfunktionen?, Tübingen I963, 5 .

45 So in einer von H. J. WolfF betreuten Dissertation: J. Terrahe, Die Beleihung als Rechtsinstitut der Staatsorganisation, Diss. jur. Münster I96I, 62 ff.

46 H. J. WolfF, O. Bachof, Verwaltungsrecht II, 4. Aufl. München I976, $\mathbb{S}$ I04. 
zweiten Hälfte des 20. Jahrhunderts zu jenem Rechtsinstitut, in dem sich auf dogmatisch überzeugende Weise ein Großteil jener Fälle unterbringen ließ, in denen der Staat öffentliche Aufgaben an Private übertrug.

Dies war sie jedoch zu Beginn des 20. Jahrhunderts nicht. Parallel zur Debatte um die Verleihung entwickelten sich andersartige - allerdings nicht unbedingt alternative oder konkurrierende, weil nicht oder nur teilweise dieselben Fallgruppen betreffende Ansätze, mit denen man die Indienstnahme Privater zu erfassen suchte. I9I I unternahm es Triepel, im Ansatz eine Systematik auszuarbeiten, die bestimmte Tätigkeitskomplexe unter dem Gesichtspunkt ihrer Einbeziehung in staatliche Gemeinwohlrealisierung unterschied: den unmittelbaren Staatsdienst durch Beamte am einen Ende der Skala, die privatrechtliche Indienstnahme durch Vertrag am anderen, dazwischen die öffentlich-rechtliche Verpflichtung zu Hilfsdiensten ohne Geschäftsbesorgungscharakter (Militärdienst, Zeugenpflicht), die Betrauung mit der Führung öffentlicher Ämter (Schöffe, kommunale Ehrenämter), den »nichtamtlichen öffentlichen Auftrag « (Vormund, Pfleger, Nachlassverwalter) und den »staatlich gebundenen Beruf « (Rechtsanwälte, Ärzte, Privatdozenten). ${ }^{47}$ Im Detail vertieft und mit übergreifenden Überlegungen zu Rechtsfolgen verknüpft hat Triepel nur die letztgenannte Fallgruppe. Aber es werden Umrisse einer systematisch-begrifflichen Erfassung sichtbar, die über den Beleihungstatbestand hinauswies, ${ }^{48}$ oder besser: eine andere und umfassendere Perspektive anbot. Allerdings fehlte es an systematischer Konsistenz, weil sich Triepels Darstellung zwischen der Auflistung von Übertragungshandlungsformen (»nichtamtlicher öffentlicher Auftrag «) und Statusbeschreibungen (»Halbbeamte«) bewegte.

Den Versuch einer systematischen Erfassung der Handlungsformen hatte Triepel aber kurz zuvor unternommen, auch wenn diese Arbeit erst Jahrzehnte später, in den Freiräumen des (zunächst halberzwungenen) Ruhestands, zu einer Publikation ausgearbeitet wurde. ${ }^{49}$ In »Delegation und Mandat « vertiefte er im Wege einer Begriffsstudie zwei Handlungsformen, über die staatliche Hoheitsgewalt übertragen wurde: die Delegation als Übertragung von Zuständigkeiten an ein anderes Subjekt, welches diese in eigenem Namen wahrzunehmen hatte, das Mandat als Rechtsgeschäft, welches die Ausübung eines fremden Amtes (in fremdem Namen) regelt. ${ }^{\circ}$ Hierbei handelte es sich um Rechtsfiguren mit übergrei-

47 H. Triepel, Staatsdienst und staatlich gebundener Beruf, in: Festschrift für Karl Binding, Bd. 2, Leipzig I9I I, 3-85.

48 Eine Teilüberschneidung mit der Verleihung kann nur insofern gesehen werden, als die Figur des Privatdozenten (später) beispielhaft für eine Tätigkeit unter Verleihungsvorbehalt genannt wurde, JeLlineK, Verwaltungsrecht (Fn. 29) 528.
49 H. Triepel, Delegation und Mandat im öffentlichen Recht, Stuttgart, Berlin I942, dort S. III zu den Entstehungsbedingungen. 50 Triepel, Delegation (Fn. 49) 23, 80. 
fender Geltungsreichweite, da sie sowohl Kompetenz- und Aufgabenverschiebungen innerhalb des staatlichen Sektors als auch im Verhältnis zwischen Staat und Privaten abdeckten. Von dieser begrifflichen Grundlage ausgehend, konnte Triepel zwischen echten Delegationen an Private (Überlassung der Polizeigewalt an eine Eisenbahngesellschaft) und unechten - weil keine tatsächlich vorhandene Kompetenz des Staates verschiebend (Anerkennung der Polizeigewalt des Kapitäns auf hoher See) - unterscheiden sowie beim Mandat zwischen Aufträgen (Bestellung eines Konkursverwalters, Lehrauftrag an Privatdozenten) und Bevollmächtigungen (denen aber oft ein Auftrag zugrundeliegt) differenzieren. ${ }^{5 \mathrm{I}}$ Erfasst wurden damit sowohl Fälle, die ansonsten als Verleihungstatbestände aufgeführt worden waren, als auch solche, die noch keine entsprechende Aufmerksamkeit gefunden hatten. Delegationen als Änderungen der rechtlichen Zuständigkeitsordnung mussten gesetzlich geregelt werden, für Mandate genügte eine Regelung durch Verwaltungsakt. ${ }^{52}$ Auch wenn sich Spuren der Triepel'schen Ordnungsbildung im heutigen Beleihungsrecht wiederfinden (Beleihung mit einer Zuständigkeit und den damit verbundenen Befugnissen nur durch Gesetz), konnte sie sich doch nicht für die Erfassung des Verhältnisses zwischen Staat und Privaten durchsetzen. Delegation und Mandat wurden zu Handlungsformen der Aufgabenverschiebung im innerstaatlichen Bereich. ${ }^{53}$

Auch mit der Entwicklung weiterer öffentlich-rechtlicher Begriffe und Institutionen vermochte man zwar Aufgabenübertragungen an Private zu erfassen, sie aber nicht eigenständig zu konturieren. So konnte Jellinek diese in sein System subjektiver öffentlicher Rechte integrieren - als subjektive Rechte auf die Übertragung bzw. die Ausübung eines Amtes, teilweise in Form des status activus, teilweise in Form des status positivus - und sie von den bloßen Reflexen objektiv-rechtlicher Befugniszuweisungen abgrenzen. ${ }^{54}$ Aber um eine spezifisch auf die Inanspruchnahme Privater zugeschnittene dogmatische Form handelte es sich nicht. Gleiches gilt für den öffentlich-rechtlichen Vertrag, dessen Anerkennung sich trotz der ablehnenden Haltung Mayers durchsetzte. ${ }^{55}$ Auch bei diesem erkannte man durchaus an, dass öffentliche Aufgaben Gegenstand einer vertraglichen Regelung sein konnten ${ }^{56}$ schließlich war Ausgangspunkt der Debatte um den öffentlich-rechtlichen Vertrag der Streit um die einseitig-hoheitliche oder vertragliche Begründung des Beamtendienstverhältnisses. ${ }^{57}$ Letzt-

5I Triepel, Delegation (Fn. 49) Ioof., I $33 \mathrm{ff}$.

52 Triepel, Delegation (Fn. 49) $28 \mathrm{f}$., I 33 .

53 Siehe schon E. Rasch, Die staatliche Verwaltungsorganisation, Köln u. a. I967, I70 ff.

54 JellineK, System (Fn. I3) $234 \mathrm{ff}$. (unter der Abschnittsüberschrift »Ausübung staatlicher Hoheitsrechte durch Private $\ll)$.
55 Dazu M. Stolleis, Geschichte des öffentlichen Rechts in Deutschland, Bd. 2, München I992, 4I 2 f. 56 So z. B. JellineK, System (Fn. I3) 249.

57 Siehe dazu W. Pakeerut, Die Entwicklung der Dogmatik des verwaltungsrechtlichen Vertrages, Berlin 2000, $20 \mathrm{ff}$. 
lich spielte diese Fallgruppe aber keine Rolle, vor allem dann, wenn man die Zulässigkeit eines öffentlich-rechtlichen Vertrages von einer gesetzlichen Erlaubnisnorm abhängig machte ${ }^{58}$ und das positive Recht wenig Anschauungsmaterial bot. Zudem bewog wo vorhanden - die äußerliche Vertragsform die Rechtswissenschaft noch lange nicht zur Annahme einer vertraglichen Regelung - so bei den mitunter in der Amts- und Gesetzessprache so bezeichneten »Konzessionsverträgen « des Eisenbahnwesens, die teilweise als Verwaltungsakte eingestuft wurden. ${ }^{59}$

III. Wegmarken der Integration der kassenärztlichen

Aufgabenwahrnehmung in einen öffentlich-rechtlichen

Funktionszusammenhang

Festzuhalten bleibt also, dass Versuche, die Überantwortung öffentlicher Funktionen an Private zu umschreiben und zu systematisieren, verschiedene Begrifflichkeiten und Institute mit unterschiedlichen Geltungsreichweiten und dogmatischen Funktionen hervorgebracht haben. Eine konzise Erfassung staatlicher Beauftragung Privater gelang aber nicht. Das war auch der Befund der Literatur nach I945, die sich wiederum an neuen Ordnungsmodellen versuchte. ${ }^{60}$ An dieser Stelle soll der Bogen wieder zu den Ausführungen des ersten Abschnitts geschlagen werden. Die Schilderung der weiteren Entwicklung des rechtlichen Verhältnisses zwischen Kassenärzten und Krankenkassen soll zeigen, auf welchen Pfaden sich unterhalb der Aufmerksamkeitsschwelle einer Rechtswissenschaft, die letztlich immer noch auf die Trennung von öffentlich-rechtlich und privatrechtlich fixiert war, die Inanspruchnahme Privater für öffentliche Aufgaben vollzog. Dabei soll die schon andernorts ${ }^{6 \mathbf{I}}$ geschilderte Entwicklung des kassenärztlichen Versorgungssystems nur insoweit in den Blick genommen werden, wie sie Effekte in Bezug auf die statusmäßige Ausgestaltung der Arztfunktion erzeugte.

Die ursprüngliche Konstruktion war - siehe oben - rein privatrechtlich: ein Dienstvertrag, der eine Geschäftsbesorgung zum Gegenstand hatte, deren Beurteilung sich wiederum am Auftragsrecht auszurichten hatte. Diese nur zwischen Kasse und Arzt bestehende Rechtsbeziehung barg indes Machtasymmetrien, denn die Krankenkasse konnte aufgrund ihres "Nachfragemonopols ${ }^{62}$ die Be-

58 Zum Beispiel Fleiner, Institutionen (Fn. 27) 202; KaUfManN, Verwaltung (Fn. I4) 7I 5; s. dazu auch H. MAurer, Der Verwaltungsvertrag - Probleme und Möglichkeiten, in: Deutsches Verwaltungsblatt $1989,798-807$, 800 , Fn. 24 m. w. N.

59 Kormann, System (Fn. 28) 39 ff.

60 H.P. IPSEN, Gesetzliche Indienstnahme Privater für Verwaltungsaufgaben, in: Um Recht und
Gerechtigkeit. Festgabe für Erich Kaufmann zu seinem 70. Geburtstage, Stuttgart, Köln I950, I4 II6I; H. GAuse, Die öffentliche Indienststellung Privater als Rechtsinstitut der Staatsorganisation, Diss. jur. Kiel 1967, insb. $49 \mathrm{ff}$.; für die NS-Zeit zu nennen ist W. Weber, Die Dienst- und Leistungspflichten der Deutschen, Hamburg I943, als Versuch der ordnenden Aufarbeitung des
Rechtsstoffs, allerdings mit dem durchgehend erkennbaren Bemühen, die systematisierenden Grundaussagen aus nationalsozialistischem Gedankengut herzuleiten.

6I KLETTE, Kassenarztverträge (Fn. 4); TAuchnitz, Die »organisierte Gesundheit (Fn. 7); D. Boerner, Normenverträge im Gesundheitswesen, München 2003.

62 Th. Gerst, Ärztliche Standesorganisation und Standespolitik in Deutschland I945-I955, Stuttgart 2004, I4. 
63 Hess / Venter, Kassenarztrecht (Fn. 4) 20; TAuchnitz, Die »organisierte" Gesundheit (Fn. 7) Io8 $\mathrm{f}$.

64 Anschaulich C. Huerkamp, Der Aufstieg der Ärzte im I9. Jahrhundert, Göttingen I985, $217 \mathrm{f}$.

65 Der Entwurf der Reichsversicherungsordnung von I9I I enthielt u. a. Bestimmungen zu einem Kollektivvertrag und Vertragsausausschüssen, die Übernahme dieser Vorschriften wurde jedoch in der zweiten Lesung abgelehnt, F. Hoffmann, Reichsversicherungsordnung, Zweites Buch, 2. Aufl. Berlin I9I 2, 458.

66 M. StOlleis, Geschichte des Sozialrechts in Deutschland, Stuttgart 2003, 79; BOERNER, Normenverträge (Fn. 6I) 24.

67 Das die neue Form der Koordinierung tragende Regelwerk wurde trotz der intensiven Beteiligung staatlicher Stellen letztlich als privatrechtlicher Vertrag anerkannt, KLETTE, Kassenarztverträge (Fn. 4) 74 .

68 Hess / Venter, Kassenarztrecht ָั (Fn. 4) 24.

dingungen diktieren. ${ }^{63}$ Die politische und wirtschaftliche Brisanz dieses Machtungleichgewichts wuchs in dem Maße, wie sich die kassenärztliche Versorgung als »Massengeschäft « etablierte und die Ärzte auf die Zuweisung von Kassenpatienten angewiesen waren. ${ }^{64}$ Im Jahre 1900 organisierten sich die Kassenärzte im Leipziger Verband (später Hartmannbund). Dessen Hauptforderung war die Ersetzung des Individualvertrages zwischen einzelnem Arzt und der Kasse durch Kollektivvereinbarungen. Der Versuch, dies in der Reichsversicherungsordnung von I9I I gesetzlich zu verankern, scheiterte allerdings. ${ }^{65}$ Stattdessen entwickelte sich nach Streikdrohungen der Ärzte und auf Druck der Regierung ${ }^{66}$ eine Form öffentlich-privater ${ }^{67}$ "Selbstverwaltung $"{ }^{68}$ die in Gestalt des zwischen den Spitzenverbänden der Ärzte und Krankenkassen geschlossenen Berliner Abkommens von I9I3 neue normative Grundlagen schuf und die Konstruktion einer einzelvertraglichen Beziehung zwischen Arzt und einzelner Krankenkasse überspielte. Nicht mehr die Kasse entschied nunmehr über die Zulassung, sondern ein beim Versicherungsamt, also einer staatlichen Behörde, gebildeter Ausschuss aus Vertretern der Ärzteschaft und der Krankenkassen. ${ }^{69}$ Es handelte sich damit letztlich nicht mehr um eine von Vertragsparteien getroffene, sondern um eine behördliche Entscheidung.

Für die Ausgestaltung der Leistungsbeziehungen zwischen Arzt und Kasse hielt man zwar zunächst noch am Modell des Einzelvertrages fest. Letztlich aber handelte es sich aus zwei Gründen um eine Fiktion. Denn erstens oblag die Vorbereitung der Verträge einem aus Vertretern der Kassen und der bereits zugelassenen Ärzte paritätisch gebildeten »Vertragsausschuß «. Dieser Ausschuss hatte den Wortlaut des Vertrages » festzustellen «. Einigten sich Arzt oder Kasse nicht auf der vom Ausschuss vorgeschlagenen Grundlage, so hatte ein Schiedsamt endgültig zu entscheiden. ${ }^{70}$ Zweitens schlossen sich in Vorwegnahme der zu erwartenden Stellungnahme des Vertragsausschusses Kassen und Ärzte auf bezirklicher Ebene zusammen, um kollektive Übereinkommen zu schließen - obwohl das Berliner Abkommen solche an sich nicht kannte. ${ }^{7 \mathbf{I}}$

Gesetzlich verankert und zugleich noch stärker behördlich organisiert - und damit in eine öffentlich-rechtliche Selbstverwaltung überführt ${ }^{72}$ - wurde diese Konstruktion I923, nachdem Einigungsbemühungen zwischen den Verbänden um eine vertragliche Fortführung des Berliner Abkommens gescheitert waren und

69 Abkommen zwischen den Organisationen der Ärzte und Krankenkassen, betreffend das Verhältnis zwischen Ärzten und Krankenkassen, vom 23. Dezember I9I3, Beilage Nr. 5 des Ministerialblatts der Handels- und Gewerbeverwaltung I9I4, $85 \mathrm{ff}$.

70 Dieses Schiedsamt bestand aus dem Vorsitzenden des Oberversicherungsamts, zwei weiteren von ihm ernannten Unparteiischen und je drei Beisitzern aus den Reihen der Ärzte und Krankenkassen.

7 I KLETTE, Kassenarztverträge (Fn. 4) 85 .

72 F. Tennstedt, Soziale Selbstverwaltung. Geschichte der Selbstverwaltung in der Krankenversicherung, Bd. 2, Bonn I977, I27; TAuchnitz, Die »organisierte « Gesundheit (Fn. 7) 30I.

73 TAuchnitz, Die »organisierte « Gesundheit (Fn. 7) 299. 
wieder ausgedehnte Ärztestreiks drohten. ${ }^{73}$ Mit der Verordnung von 1923 wurde der Kollektivvertrag endgültig anerkannt und einer stärkeren amtlichen Einflussnahme unterstellt. Die Vertragsschließenden, also die Verbände der Kassen und Kassenärzte, hatten sich den Richtlinien des Reichsausschusses der Ärzte und Krankenkassen ${ }^{74} \mathrm{zu}$ unterwerfen, ${ }^{75}$ welche auch bald darauf ergingen. ${ }^{76}$ Die Regelungswirkung des an sich noch maßgeblichen Einzelvertrags reduzierte sich faktisch auf die individuelle Anerkennung des Kollektivvertrags. ${ }^{77}$ Auch in anderer Hinsicht war der privatrechtlich-dienstrechtliche Charakter des Arzt-Kassen-Verhältnisses durch öffentlich-rechtliche Bestimmungen unterhöhlt: Ausführungsvorschriften des Reichsarbeitsministeriums geboten die Weiterführung der ärztlichen Tätigkeit auch bei Kündigung des Dienstvertrages. ${ }^{78}$

Die Notverordnung vom 8. Dezember I93 I - ebenfalls eine aus dem Scheitern von Vertragsverhandlungen resultierende, aber weitgehend auf verbandlichen Vorarbeiten beruhende ultima ratio $^{79}$ - beseitigte dann endgültig die Relevanz des Einzelvertrages: Die Zulassung erfolgte für alle Kassen. Dass der Einzelvertrag letztlich nur die Beitrittserklärung zum Kollektivvertrag war, war jetzt auch gesetzlich festgeschrieben. ${ }^{80}$ Das nationalsozialistische Kassenrecht bekräftigte dann lediglich die öffentlich-rechtliche Bindung des Kassenarztes ${ }^{8 \mathbf{I}}$ - eine privatrechtliche Rechtsbeziehung des Arztes zur Kasse bestand nicht mehr $^{82}$ - und zentralisierte das Verbandswesen. ${ }^{83}$ Die Verbände wurden - wie von den Kassenärzten schon vorher angestrebt ${ }^{84}$ - in öffentlich-rechtliche Körperschaften umgewandelt.

Die Gesetzgebung nach 1945 konnte an der I93 I geschaffenen Ordnung anknüpfen. ${ }^{85}$ Aus dem noch I 886 so beschriebenen ${ }^{86}$ privatrechtlichen (im Rahmen eines Dienstvertrages von der Kasse erteilten) Auftrag war der »öffentlich-rechtliche Auftrag « der öffentlich-rechtlichen Kassenärztlichen Vereinigung zugunsten des Versicherten und zu Lasten der Kasse ${ }^{87}$ geworden und aus der privatrechtlichen Dienstverpflichtung des Arztes gegenüber der Kasse eine dem Kassenarzt obliegende öffentlich-rechtliche Verpflichtung gegenüber der Kassenärztlichen Vereinigung. ${ }^{88} \mathrm{Die}$ Ausgestaltung der ärztlichen Funktion war in die Herrschaft des Verbandssystems übergegangen. ${ }^{89}$ Bei dieser Konstruktion verblieb es im Wesentlichen bis heute. ${ }^{\circ 0}$

74 Dieser setzte sich zusammen aus je fünf Vertretern der beiden Gruppen sowie drei vom Reichsarbeitsminister ernannten Mitgliedern.

$75 \llbracket 5$ Verordnung über Ärzte und Krankenkassen vom 30. Oktober I923 (RGBl. I S. IO5 I).

76 Richtlinien für den allgemeinen Inhalt der Arztverträge, aufgestellt von dem Reichsausschuß für Ärzte und Krankenkassen, am I2. Mai I924 und später (abgedr. in:
L. Sievers, Handbuch der Kassenärzte, 2. Aufl., Hannover I925, I 7 ff.); Bestimmungen über das Verfahren bei der Zulassung zur Kassenpraxis (Zulassungsbestimmungen, Reichsausschuß I 5 . Mai I925) (abgedr. bei SIEvers a.a. O., 3 I ff.).

77 Hess / Venter, Kassenarztrecht (Fn. 4) 28.

78 (red.), Dürfen die Ärzte streiken?, in: Der Kassenarzt 6/I925, 5 .
79 Tauchnitz, Die »organisierte « Gesundheit (Fn. 7) 2 I8.

80 I I Abs. 3 V. Teil, Kap. I, Abschn. I, Vierte Verordnung des Reichspräsidenten zur Sicherung von Wirtschaft und Finanzen und zum Schutz des inneren Friedens vom 8. Dezember I93 I (RGBl. I S. 699).

8I $\int$ I lit. c Verordnung über die Zulassung von Ärzten zur Tätigkeit bei den Krankenkassen vom I7. Mai I934 (RGBl. I S. 399): Durch die Zulassung wurde eine Verpflichtung des Arztes gegenüber der Kassenärztlichen Vereinigung zur Teilnahme an der kassenärztlichen Versorgung begründet. Die Zulassung erfolgte gem. $\int 33$ nunmehr durch einen Zulassungsausschuss, dessen Mitglieder vom Reichsführer der Kassenärztlichen Vereinigung zu berufen waren.

82 F. Hoffmann, J. Kreil, Kommentar zur Reichsversicherungsordnung, Berlin I939, $\mathbb{\int} 368$ Pkt. IV.

$83 \sqrt{ }$ I Abs. I Verordnung über die Kassenärztliche Vereinigung Deutschlands vom 2. August 1933 (RGBl. I S. 567) (Kassenärztliche Vereinigung Deutschlands wird Träger der Beziehungen zwischen Kassen und Kassenärzten); $\mathbb{\int} 87$ Reichsärzteordnung I3. Dezember I935 (RGBl. I S. I433) (Auflösung des Hartmannbundes und Bestimmung der Kassenärztlichen Vereinigung Deutschlands als Rechtsnachfolgerin); Art. I Zwölfte Verordnung zur Neuordnung der Krankenversicherung vom 6. September I937 (RGBl. I S. 964) (Bildung zentralisierter Reichskassenverbände).

84 Gerst, Ärztliche Standesorganisation (Fn. 62) I 5 .

85 BoERner, Normenverträge (Fn. 6I) 27.

86 Siehe oben I.

87 Hess / Venter, Kassenarztrecht (Fn. 4) I I 5 .

88 Tauchnitz, Die »organisierte « Gesundheit (Fn. 7) 220.

89 So i. E. auch M. STOlleis, Hundert Jahre Gesetzliche Krankenversicherung, in: Zeitschrift für Sozialreform 9/10/I983, 6I 2$623,617$.

90 Siehe nur A. FLÜCHTER, Kollektivverträge und Konfliktlösung im SGB V, Baden-Baden 2000, 26. 
IV. Schlussüberlegungen

Der Blick auf die Entwicklung der rechtlichen Stellung des Kassenarztes soll Folgendes verdeutlichen: Erstens hat man es mit einem Zusammenspiel von Elementen der Indienstnahme Privater zu tun, die begrifflich und rechtssystematisch unterschiedlich einzuordnen sind. Das Institut des Auftrags machte einen Gestaltwandel durch - von einem privatrechtsdogmatisch recht unproblematisch zu erfassenden Geschäftsbesorgungsvertrag zu einem wenig konturierten öffentlich-rechtlichen Mandat in Form einer gegenüber der Kassenärztlichen Vereinigung bestehenden Verpflichtung zur Teilnahme an der gesundheitlichen Versorgung; Anklänge an das Triepel'sche Mandat finden sich hier durchaus, auch wenn der Fall in seiner zu Jahrhundertbeginn entstandenen Darstellung natürlich noch nicht aufgeführt werden konnte. Auch in der von Triepel entworfenen Figur des staatlich gebundenen Berufs mochte sich der Kassenarzt wiederfinden - mit all den von Triepel herausgearbeiteten rechtlichen Konsequenzen: rechtlich definierter Aufgabenbereich, Festsetzung beruflicher Pflichten, Bindung an (in Dienstanweisungen, aber auch Kammervorschriften statuierte) Maßstäbe ordnungsgemäßer Berufsausübung, (mittelbare) staatliche Beaufsichtigung.

Ferner lässt sich dem Status des Kassenarztes auch ein subjektives Recht auf Ausübung einer öffentlichen Aufgabe im Sinne Jellineks entnehmen, denn die zur Übernahme der kassenärztlichen Versorgung berechtigende Zulassung konnte nur unter besonderen Voraussetzungen entzogen werden und die Zulassung wiederum begründete ein Recht auf Vertragsabschluss. ${ }^{9 \mathrm{I}}$ Weiter setzte sich die Auffassung durch, dass die Kollektivvereinbarungen - nachdem die vertragsschließenden Verbände einen öffentlich-rechtlichen Status zuerkannt bekommen hatten - öffentlich-rechtliche Verträge darstellten. ${ }^{92}$ Und schließlich wurde auch immer wieder diskutiert, den Kassenarzt als Beliehenen einzustufen. ${ }^{93}$

In der Entwicklung des Kassenarztstatus spiegelt sich eine Vielzahl rechtsdogmatischer Systematisierungstopoi. Verallgemeinernd kann man also sagen, dass sich die Inanspruchnahme Privater für öffentliche Aufgaben nicht nur im Wege der Zuordnung $\mathrm{zu}$ einzelnen rechtswissenschaftlich entwickelten Figuren herausarbeiten lässt. Vielmehr bedarf es - was hier nur ansatzweise geschehen konnte - mehrerer Reflexionsschritte: eines Befundes des

9I BoERner, Normenverträge (Fn. 6I) 24.

92 KLETTE, Kassenarztverträge (Fn. 4) I67 ff., I99.

93 Siehe dazu F.E. Schnapp, Muss ein Vertragsarzt demokratisch legitimiert sein?, in: Neue Zeitschrift für Sozialrecht 200I, 33734I, 339 (selbst den Beliehenenstatus ablehnend). 
zeitgenössisch vorhandenen Bestandes an Rechtsfiguren und Systematisierungsmustern, der Herausarbeitung vorhandener - gesetzlicher und untergesetzlicher - Regelungsarrangements, der Suche nach rechtsdogmatischer Erfassung dieser Regelungsarrangements - und damit der Feststellung zeitgenössischer Aufmerksamkeitsdefizite. Deutlich wird dabei auch der Vorsprung der unablässig eine Vielzahl neuer Formen hervorbringenden interventionsstaatlichen Normenproduktion vor der seinerzeitigen Dogmatik, die den außerhalb des klassischen Eingriffsrechts liegenden Materien ohnehin weniger Aufmerksamkeit widmete. ${ }^{94}$

Zweitens sollen die Ausführungen deutlich machen, dass die Inanspruchnahme Privater nicht nur in einem zweipoligen Verhältnis zwischen Staat und Beauftragten erfolgte, sondern oft durch zwischengeschaltete privatrechtlich oder öffentlich-rechtlich organisierte intermediäre Verbände vermittelt wurde. Diese waren allerdings keine neutralen Makler, sondern drückten den betreffenden Aufgabenkomplexen durch eigene Normsetzung und Normdurchsetzung ihren Stempel auf. Dies gilt für die zunächst privatrechtlich, dann öffentlich-rechtlich organisierten Dachvereinigungen von Kassenärzten und Kassen, aber auch für die Ärztekammern, die die Kassenärzte mittels ihrer Disziplinargewalt schon vor der Kollektivvertragsregelung am Abschluss »lohndrückerischer " Verträge gehindert hatten. ${ }^{95}$ Im Hinblick auf diese selbstregulative Prägung privater Aufgabenerfüllung durch Verbände ist ebenfalls ein Aufmerksamkeitsdefizit der zeitgenössischen Rechtswissenschaft zu konstatieren. ${ }^{96}$

Drittens wird die Gesetzesabhängigkeit der literarischen Hervorbringung von Rechtsfiguren erkennbar. Soweit die Literatur Ordnungsüberlegungen zur Indienstnahme Privater anstellte, entnahm sie ihr Anschauungsmaterial oft der Gesetzgebung. Dies ist an sich auch unabdingbar für die Arbeitsweise der Rechtsdogmatik. Jedoch erfasste sie damit nur einen Teilausschnitt. Normativ konturiert wurde der Status des Kassenarztes zu jener Zeit, in der er an sich - wenn man nur auf die gesetzlichen Regelungen abstellt - noch vollständig dem Privatrechtsregime unterlag, durch verbandliche Normsetzung. Schon vor dem Berliner Abkommen von I9I3 hatte die untergesetzliche Normierung der Art und Weise der kassenärztlichen Aufgabenerfüllung eingesetzt - z. B. durch administrative Normalstatuten für die Krankenkassen, ${ }^{97}$ durch Selbstkoordinierung der Ärzteschaft ${ }^{98}$ oder durch regional

94 Siehe dazu M. Stolleis, Die Entstehung des Interventionsstaates und das öffentliche Recht, in: ZNR I I (I989) I29-I46, I42f.

95 TAuchnitz, Die »organisierte « Gesundheit (Fn. 7) 2 I2.

96 So auch in aller Eindringlichkeit F. GLum, Der deutsche und der französische Reichswirtschaftsrat. Ein Beitrag zu dem Problem der Repräsentation der Wirtschaft im Staat, Berlin, Leipzig I929, 68.
$97 \int 24$ des Normalstatuts für die Krankenkassen vom I4. März I 884, Quellensammlung (Fn. I) Anhang A, gab z. B. dem Kassenvorstand auf, Leitlinien für die Verschreibung von Arzneimitteln zu erlassen. - Allerdings beanspruchte das Normalstatut keine unbedingte Verbindlichkeit, sondern enthielt Empfehlungen; siehe Pkt. I der Vorbemerkungen des Normalstatus.
98 Bestimmungen für die ärztlichen Verordnungen bei den Kassen mit freier Arztwahl zu Berlin, hg. vom Vorstand der freigewählten Kassenärzte zu Berlin und Umgebung, Berlin I 896. - Diese Richtlinien hatten (6) nur empfehlenden Charakter; es kann jedoch davon ausgegangen werden, dass sie in großem Umfang befolgt wurden. 
99 So z. B. schon I 892 für Düsseldorf und Berlin; vgl. Tauchnitz, Die »organisierte« Gesundheit (Fn. 7) 217.

ฐั

Im Auftrag der Kasse begrenzte Vereinbarungen zwischen Kassen und lokalen Ärztevereinen. ${ }^{99}$ Das Berliner Abkommen hatte dann ein umfassendes Regelwerk $^{\text {100 }}$ geschaffen, welches neuartige Ordnungsbildungen stimulierte. Zwar ließen sich diese Regelungen (bis auf die Normalstatuten) noch als privatrechtliche Verträge einstufen, aber durch Beteiligung der Regierung am Zustandekommen, durch Unterwerfung unter ein staatliches Aufsichtsrecht oder durch behördliche Beteiligung an der Durchsetzung (Schiedsgerichte) waren der staatlichen Seite umfassende Ingerenzmöglichkeiten eröffnet; auf dem sicheren Boden des Privatrechts befand man sich nicht mehr. Untergesetzliche Normierung konkretisierte also nicht nur Gesetzesrecht, sondern etablierte teilweise parallele - nicht dem Gesetz konträre, aber gesetzliche Grundentscheidungen erheblich modifizierende - Ordnungen oder nahm gesetzliche Gestaltung vorweg.

Viertens wird, jedenfalls in Bezug auf einzelne Rechtsinstitute, die Mehrstufigkeit von Transferprozessen sichtbar. Die Verleihung eines öffentlichen Unternehmens im Sinne Otto Mayers fußte auf dem Modell der französischen concession de travaux publics, dieses wiederum eingebettet in das Konzept des service public einer andersartigen Form des staatlichen Zugriffs auf die Gestaltung der öffentlichen Geschäfte. ${ }^{\text {IOI }}$ Rezipiert wurde das Modell zwar auch in der deutschen Verwaltungsrechtsliteratur, jedoch schon in Weimar partiell ersetzt durch Rückbindungselemente zur deutschen Rechtstradition; in der Bundesrepublik war dann die Verbindung zur Mayer'schen französischrechtlichen Grundlage endgültig gekappt. Es handelte es sich somit um einen Vermittlungsprozess, in dem der Transfer zwischen nationalen Rechtsordnungen letztlich absorbiert wurde durch sich anschließende nationalstaatliche rechtsdogmatische Weiterentwicklungen.

Fünftens schließlich zeigt sich, dass das für die Entstehung des Interventionsstaates charakteristische Vordringen öffentlich-rechtlicher Formen ${ }^{\mathrm{IO} 2}$ nicht nur eine vertikale, also die Beziehung zwischen Staat und Bürger betreffende, Spielart aufweist, sondern auch eine horizontale: In Verteilungskämpfen liegende gesellschaftliche Interessengruppen entdeckten die Vorteile des öffentlichen Rechts sei es, dass sich in öffentlich-rechtlichen Organisationen ihre Geltungsansprüche offensiver vortragen ließen, sei es, dass sich durch öffentlich-rechtliche Regelung ihrer Beziehungen gegenseitige Blockaden auflösen ließen, sei es, dass sie ihre Berufsgruppen durch
Ioo Bestandteil des Regelungspakets waren nicht nur das eigentliche Abkommen, sondern auch der Erlass des Ministers für Handel und Gewerbe vom I7. Februar I9I4, der Vorgaben für die Integration der Versicherungsämter in das neue System enthielt, Bestimmungen über die Bildung des Schiedsamts, über den Zentralausschuss, Verhandlungsniederschriften, in denen einige Bestimmungen des
Berliner Abkommens verbindlich erläutert wurden, sowie Wahlordnungen für die einzelnen Organe (alle abgedruckt in: Beilage Nr. 5 des Ministerialblatts der Handelsund Gewerbeverwaltung [Fn. 69], 87 ff.).

IOI Siehe O. Mayer, Theorie des französischen Verwaltungsrechts, Straßburg I 886, 302 ff.

IO2 STOLLEIS, Interventionsstaat (Fn. 94) I36 ff. 
Zuerkennung eines halbamtlichen Status mit einer Gemeinwohlaura schmücken konnten.

Mit welchen rechtskonstruktiven Lösungen man seinerzeit die hoheitliche Inanspruchnahme Privater zu erfassen suchte, lässt sich somit nur nachvollziehen, wenn man den Blick nicht nur auf einzelne, sondern auf eine Vielzahl nebeneinander entwickelter dogmatischer Lösungsansätze richtet. Und es sind auch jene in der Praxis entstandenen Modelle zu berücksichtigen, die von der Rechtsdogmatik zwar gelegentlich registriert, aber nicht in die Begriffs- und Systementwicklung transformiert wurden. ${ }^{\text {I03 }}$ Vielleicht lässt sich auf diesem Wege ein »Rechtssystem « rekonstruieren, welches ein historisch aussagekräftiges Bild von der Funktionsweise des Rechts vermittelt.

Peter Collin

I03 Siehe die etwas farblose Charakterisierung bei L. Richter, Sozialversicherungsrecht, Berlin I93 I, 223, der konstatierte, dass sich die Beziehung zwischen Arzt und Kasse grundsätzlich nach privatrechtlichem Dienstrecht richtet.

Aber: »Um diese privatrechtlichen Beziehungen ranken sich dann wieder besondere öffentlichrechtliche Vorschriften." 